Research Paper

\title{
The association between urinary Alzheimer-associated neuronal thread protein and cognitive impairment in late-life depression: a controlled pilot study
}

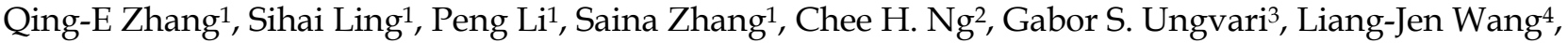 \\ Sheng-Yu Lee ${ }^{5}$, Gang Wang ${ }^{\bowtie}$, Yu-Tao Xiang ${ }^{5}$ \\ 1. National Clinical Research Center for Mental Disorders \& Beijing Key Laboratory of Mental Disorders, Beijing Anding Hospital, Capital Medical University, \\ Beijing, China \\ 2. Department of Psychiatry, University of Melbourne, Melbourne, Victoria, Australia \\ 3. The University of Notre Dame Australia / Graylands Hospital, Perth, Australia \\ 4. Department of Child and Adolescent Psychiatry, Kaohsiung Chang Gung Memorial Hospital, Chang Gung University College of Medicine, Kaohsiung, \\ Taiwan \\ 5. Unit of Psychiatry, Faculty of Health Sciences, University of Macau, Macao SAR, China
}

$\square$ Corresponding authors: Yu-Tao Xiang, 3/F, Building E12, Faculty of Health Sciences, University of Macau, Avenida da Universidade, Taipa, Macau SAR, China. Fax: +853-2288-2314; Phone: +853-8822-4223; E-mail: xyutly@gmail.com; or Dr. Gang Wang, Mood Disorders Center, Beijing Anding Hospital, Beijing, China. E-mail: gangwangdoc@gmail.com.

(c) Ivyspring International Publisher. This is an open access article distributed under the terms of the Creative Commons Attribution (CC BY-NC) license (https://creativecommons.org/licenses/by-nc/4.0/). See http://ivyspring.com/terms for full terms and conditions.

Received: 2018.01.17; Accepted: 2018.04.21; Published: 2018.08.10

\begin{abstract}
Accumulation of tau protein is associated with both Alzheimer's disease (AD) and late-life depression (LLD). Alzheimer-associated neuronal thread protein (AD7c-NTP), which is closely linked with the tau protein, is elevated in the cerebrospinal fluid and urine of $A D$ patients. This study examined the association between urinary AD7c-NTP and late-life depression with cognitive impairment. One hundred and thirty-eight subjects were recruited into late-life depression with cognitive impairment (LLD-Cl, $n=52$ ), late-life depression without cognitive impairment (LLD-NCl, $n=29), A D(n=27)$, and healthy control $(H C, n=30)$ groups. The level of urinary AD7c-NTP was measured using the enzyme-linked immunosorbent assay method. The Montreal Cognitive Assessment scale (MoCA), Hamilton Rating Scale for Depression (HRSD) and Hamilton Anxiety Rating Scale (HAMA) were used to assess cognitive functions and depressive and anxiety symptoms in the AD and LLD groups. Urinary levels of AD7c-NTP in the LLD-Cl group $(1.0 \pm 0.7 \mathrm{ng} / \mathrm{ml})$ were significantly higher than both the LLD-NCl $(0.5 \pm 0.3 \mathrm{ng} / \mathrm{ml})$ and $\mathrm{HC}$ groups $(0.5 \pm 0.3 \mathrm{ng} / \mathrm{ml})$, but lower than in the AD group $(1.6 \pm 1.7$ $\mathrm{ng} / \mathrm{ml})$. No significant associations were found in the level of urinary AD7c-NTP in relation to age, gender, education and MoCA in the LLD-Cl group. The level of urinary AD7c-NTP appears to be associated with cognitive impairment in late-life depression and may be a potential biomarker for early identification of cognitive impairment in LLD.
\end{abstract}

Key words: AD7c-NTP, Alzheimer's disease, late-life depression, cognitive impairment

\section{Introduction}

Late-life depression (LLD), defined as major depression occurring in older adults regardless of the age of onset [1], is common (10\%-38\%) among older people [2-4], and is associated with an increased risk of disability, suicide, high mortality and poor quality of life $[5,6]$. Cognitive impairment, such as in episodic memory, speed of information processing, executive functioning, and visuospatial ability [7-9] often occurs in LLD [10], which is associated with increased relapse rate, functional impairment and poor treatment adherence [1]. It was once thought that cognitive impairment could improve after the recovery of depression, thus the term "pseudo-dementia" was used to describe cognitive impairment in LLD [11]. However, it became evident that cognitive impairment persisted in a significant number of LLD patients even after the depressive symptoms improved [12].

In recent years the association between LLD and Alzheimer's disease (AD) has gained much attention. 
The differential diagnosis of LLD and AD can be difficult to tease out in clinical practice. On one hand, cognitive impairment in LLD could be prominent resulting in the misdiagnosis of early stage $A D$, and on the other hand, patients with incipient $A D$ may often present with somatic and mood symptoms including depression. LLD may be an early manifestation or a risk factor of $\mathrm{AD}[4,13,14]$, which is supported by epidemiological studies that found an increased risk of LLD converting to dementia compared to healthy controls [15] and possible progressive dementia within 2-4 years following depressive episodes of $\operatorname{LLD}[16,17]$.

There appears to be similarities between LLD and $\mathrm{AD}$ in terms of neuropathology and brain structure [18, 19]. For example, there are neuronal loss, $\beta$-amyloid plaques and neurofibrillary tangles (NFTs) in AD patients, especially in the hippocampus $[20,21]$. Similarly, there are also significant deposition of beta amyloid and accumulation of tau protein in the brain of LLD patients [22-24], which suggests a potential link between LLD and AD.

Early identification and treatment of cognitive impairment in LLD could have important clinical implications for dementia prevention [25, 26]. However, assessing cognitive function in LLD patients can be challenging because it is difficult to differentiate cognitive impairment from depressive symptoms, and some severely depressed older patients are unable to complete cognitive assessments. There is currently no biomarker available to accurately identify cognitive impairment in LLD [27].

Alzheimer-associated neuronal thread protein (AD7c-NTP), a transmembrane phosphoprotein, is found in central nervous system cells. Over-expression of AD7c-NTP could lead to neuritic sprouting and cell death, which is associated with pathological changes in $\mathrm{AD}[28,29]$. AD7c-NTP accumulation co-localizes with NFTs and malnutrition axons [30]. In addition, AD7c-NTP is positively associated with phosphorylated tau accumulation in central neurons and cerebrospinal fluid (CSF) in AD patients [31, 32]. Urinary AD7c-NTP level has been shown to have high sensitivity and specificity in predicting amyloid- $\beta$ deposition in AD and mild cognitive impairment (MCI) [33]. Moreover, some studies found increased AD7c-NTP concentration in cerebrospinal fluid and urine in the early course of neurodegeneration in $\mathrm{AD}$, which is positively associated with $\mathrm{AD}$ severity [34-37]. A meta-analysis has suggested that urinary AD7c-NTP may be helpful in the early diagnosis of probable AD [38].

There are few studies that have examined the level of AD7c-NTP in LLD. A longitudinal study found that the urinary AD7c-NTP level in LLD was significantly increased at 24 months compared to baseline, which was higher than in a healthy control group [39]. However, the study lacked any comparison of AD7c-NTP levels with AD patients and between LLD patients with and without cognitive impairment. Further, the study did not control for potentially confounding effects of age, gender, and education.

Hence, this study aimed to compare urinary AD7c-NTP levels in four distinct groups, including LLD with cognitive impairment (LLD-CI), LLD without cognitive impairment (LLD-NCI), AD and healthy controls $(\mathrm{HC})$, and to examine the association of urinary AD7c-NTP level in relation to age, gender, education, and cognitive function.

\section{Methods}

\section{Participants and study setting}

This case-control study was conducted between January 1, 2016 and August 31, 2017 in Beijing Anding Hospital of Capital Medical University in China.

Depressed subjects were consecutively invited to participate in the study if they fulfilled the following inclusion criteria: 1) a current episode of major depressive disorder according to the DSM-IV criteria [40] based on a clinical interview; 2) aged 55 years or older; 3) Hamilton Depression Rating Scale (HDRS17) total score of $\geq 18$ indicating moderate-severe depression; 4) ability to understand the contents of the interview. Exclusion criteria were: 1) any other psychiatric comorbidities and MCI; 2) major medical conditions; 3) use of psychotropic drugs or other medications that may affect cognition in the past two weeks. Eligible patients were further divided into two groups according to the Montreal Cognitive Assessment (MoCA) total score: (1) late-life depression with cognitive impairment group (LLD-CI; MoCA total score < 26); (2) late-life depression without cognitive impairment group (LLD-NCI; MoCA total score of $\geq 26$ ) [41, 42].

The AD group was consecutively recruited during the study period if they fulfilled the following inclusion criteria: 1) diagnosis of $\mathrm{AD}$ according to the criteria devised by the United States National Institute of Neurology Disorders and Stroke and the Alzheimer's Disease and Related Disorders Association (NINCDS-ADRDA) [43]; 2) aged 55 years or older; 3) the Chinese version of the Mini Mental State Examination (MMSE) total score of $\leq 26$ [44]; 4) HDRS-17 total score of $\leq 7$; 5) Hachinski Ischemic Score (HIS) score of $\leq 4$ [45]; 6) able to understand the contents of the interview. Exclusion criteria were: 1) cognitive dysfunction associated with neurologic 
conditions such as cerebrovascular diseases, syphilis encephalopathy, traumatic brain injury, or alcohol or drug abuse; 2) use of psychotropic drugs or other medications that may affect cognition in the past two weeks.

Healthy controls (HC) matched in age ( \pm 3 years) and education $( \pm 3$ years $)$ were recruited by advertisements from the community. Inclusion criteria were: 1 ) aged 55 years or older; 2) MMSE total score of $>26$. Exclusion criteria were: 1) past or current history of psychiatric disorder; 2) focal lesion(s) in the brain on CT or MRI scans.

The study protocol was approved by the Research and Ethics Committee of Beijing Anding Hospital. All participants provided written informed consent.

\section{Measures and assessments}

\section{Detection of urine AD7c-NTP}

Clean midstream urine specimens were collected from all subjects in the morning and immediately centrifuged, and then preserved in a refrigerator at $4^{\circ} \mathrm{C}$. The levels of AD7c-NTP in urine specimens were measured using the enzyme-linked immunosorbent assay AD7c-NTP kit (Anqun Biological Technology Co. Ltd., Shenzhen, China), which is the only kit approved for such clinical test in China. A microplate reader at $450 \mathrm{~nm}$ wavelength was used to measure absorbance (A value) and the AD7c-NTP concentration.

\section{Measures}

Demographic and clinical characteristics of all subjects were recorded on a data collection sheet via a review of medical records and confirmed in a clinical interview conducted by two attending psychiatrists. The Chinese version of the Montreal Cognitive Assessment scale (MoCA) [46, 47] was used to evaluate cognitive functions in aspects of visuospatial, executive, naming, attention, language, abstraction, delayed recall and orientation. The MoCA is a short (5-10 minutes), user-friendly cognitive screening tool for $\mathrm{AD}$ and mild cognitive impairment [46, 48]. The contents of MoCA focus more on executive function and attention than other short cognitive measures, such as the MMSE, therefore it is more suitable in detecting non-AD cognitive impairment [49]. The severity of depressive and anxiety symptoms were assessed using the Chinese versions of the HRSD-17 [50, 51], and the Hamilton Anxiety Rating Scale (HAMA) [51, 52], respectively.

\section{Statistical analysis}

Data were analyzed by the Statistical Package for Social Sciences (SPSS), Version 21.0. Chi-square tests and analysis of variance were used to compare the demographic and clinical variables and neuropsychology performances, as appropriate. The MoCA, HAMA, HRSD-17 total scores and urine AD7c-NTP levels were compared between the four groups using analysis of covariance (ANCOVA) after controlling for age, education levels and gender. The associations between urinary AD7c-NTP levels and age, gender, education, and the MoCA total score were assessed with Spearman's rank correlations. The levels of significance were set at 0.05 (two-tailed).

\section{Results}

Altogether, 138 subjects were recruited into 4 groups: LLD-CI $(n=52)$, LLD-NCI $(n=29), A D(n=30)$ and HC groups $(n=27)$. The comparisons of basic demographic and clinical characteristics between groups are shown in Table 1. There were significant group differences in MoCA total scores and all its domains (all $\mathrm{p}$ values $<0.05$ ).

Table 1. Comparison of demographic and clinical variables between groups

\begin{tabular}{|c|c|c|c|c|c|c|c|c|c|c|c|}
\hline \multirow[t]{2}{*}{ Variables } & \multicolumn{2}{|c|}{ LLD-CI (n=52) } & \multicolumn{2}{|c|}{ LLD-NCI (n=29) } & \multicolumn{2}{|c|}{$\mathrm{AD}(\mathrm{n}=30)$} & \multicolumn{2}{|c|}{$\mathrm{HC}(\mathrm{n}=27)$} & \multicolumn{3}{|c|}{ Statistics } \\
\hline & $\mathrm{N}$ & $\%$ & $\mathrm{~N}$ & $\%$ & $\mathrm{~N}$ & $\%$ & $\mathrm{~N}$ & $\%$ & $\chi^{2}$ & df & $p$ \\
\hline Male sex & 17 & 32.7 & 15 & 51.7 & 15 & 50.0 & 17 & 63.0 & 7.3 & 3 & 0.06 \\
\hline \multirow[t]{2}{*}{ Formal education $\leq 9$ years } & 26 & 50.0 & 15 & 51.7 & 19 & 63.3 & 13 & 48.1 & 1.7 & 3 & 0.63 \\
\hline & Mean & SD & Mean & SD & Mean & $\mathrm{SD}$ & Mean & SD & $\mathrm{F}$ & df & $p$ \\
\hline Age (years) & 64.2 & 6.2 & 63.1 & 5.7 & 66.5 & 6.1 & 64.5 & 5.4 & 1.7 & 3 & 0.16 \\
\hline HAMA total & 24.7 & 8.8 & 23.0 & 6.3 & & & & & 0.6 & 1 & 0.41 \\
\hline HRSD-17 total & 27.4 & 7.9 & 24.1 & 7.2 & & & & & 2.7 & 1 & 0.10 \\
\hline MoCA total & 21.5 & 3.1 & 27.3 & 1.1 & 13.8 & 4.7 & 27.5 & 1.3 & 133.0 & 3 & $<0.001$ \\
\hline Visuospatial/Executive & 3.4 & 1.2 & 4.4 & 0.6 & 2.5 & 1.2 & 4.5 & 0.6 & 24.3 & 3 & $<0.001$ \\
\hline Naming & 7.2 & 0.5 & 3.0 & 0.0 & 2.0 & 0.7 & 3.0 & 0.1 & 4.7 & 3 & $<0.001$ \\
\hline Attention & 4.8 & 1.1 & 5.8 & 0.4 & 2.2 & 1.3 & 5.7 & 0.4 & 89.4 & 3 & $<0.001$ \\
\hline Language & 1.9 & 0.8 & 2.8 & 0.3 & 1.3 & 0.9 & 2.7 & 0.5 & 29.0 & 3 & $<0.001$ \\
\hline Abstraction & 1.3 & 0.6 & 1.7 & 0.4 & 1.3 & 0.8 & 1.9 & 0.2 & 8.1 & 3 & $<0.001$ \\
\hline Delayed recall & 1.7 & 1.4 & 3.4 & 0.9 & 0.2 & 0.3 & 3.4 & 1.0 & 58.6 & 3 & $<0.001$ \\
\hline Orientation & 5.4 & 1.0 & 5.9 & 0.2 & 4.2 & 1.0 & 6.0 & 0.0 & 29.2 & 3 & $<0.001$ \\
\hline
\end{tabular}




\section{Urinary level of AD7c-NTP}

After controlling for the potentially confounding effects of age, education level, and gender, there were significant group differences in urinary AD7c-NTP levels (Table 2). Specifically, urinary AD7c-NTP levels in the LLD-CI group were significantly higher than those in the HC and LLD-NCI groups, but lower than found in the AD group (all $p$ values < 0.05).

Table 2. Comparisons of urinary AD7c-NTP levels between the LLD-Cl, LLD-NCl, AD, and HC groups

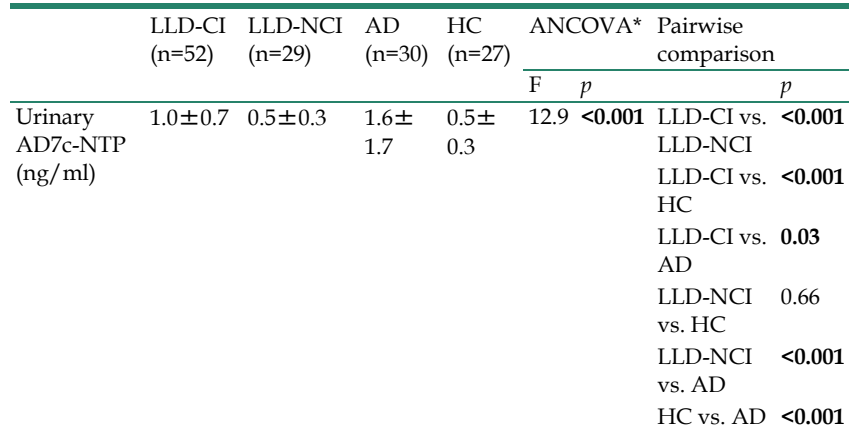

* The level of AD7c-NTP was not normally distributed. A log transformation has been used in ANCOVA. Bold values: $\mathrm{p}<0.05 ; \mathrm{AD} 7 \mathrm{c}-\mathrm{NTP}=$ Alzheimer-associated neuronal thread protein. LLD-CI=late-life depression with cognitive impairment; LLD-NCI=late-life depression without cognitive impairment; $\mathrm{AD}=$ Alzheimer's disease; $\mathrm{HC}=$ healthy control

\section{Comparison of MoCA domain between LLD-CI and AD groups}

Compared to the AD group, subjects in the LLD-CI group had significantly lower scores in visuospatial, executive, naming, attention, language, delayed recall and orientation, but not in the abstraction domains (Table 3).

Table 3. Comparisons of MoCA scores between the LLD-Cl and AD groups

\begin{tabular}{|c|c|c|c|c|c|c|c|}
\hline \multirow[t]{2}{*}{ Variables } & \multicolumn{2}{|c|}{$\begin{array}{l}\text { LLD-CI } \\
(\mathrm{n}=52)\end{array}$} & \multicolumn{2}{|c|}{$\mathrm{AD}(\mathrm{n}=30)$} & \multicolumn{2}{|c|}{ Statistics } & \multirow[b]{2}{*}{$p$} \\
\hline & Mean & SD & Mean & SD & $\mathrm{F}$ & $\mathrm{df}$ & \\
\hline MoCA total & 21.5 & 3.1 & 13.8 & 4.7 & 61.4 & 1 & $<0.001$ \\
\hline $\begin{array}{l}\text { Visuospatial/Executive } \\
\text { (range: } 0-5 \text { ) }\end{array}$ & 3.4 & 1.2 & 2.5 & 1.2 & 5.4 & 1 & 0.02 \\
\hline Naming (range: $0-3$ ) & 7.2 & 0.5 & 2.0 & 0.7 & 21.6 & 1 & $<0.001$ \\
\hline Attention (range: 0-6) & 4.8 & 1.1 & 2.2 & 1.3 & 82.1 & 1 & $<0.001$ \\
\hline Language (range: 0-3 & 1.9 & 0.8 & 1.3 & 0.9 & 7.0 & 1 & 0.01 \\
\hline Abstraction (range: 0-2) & 1.3 & 0.6 & 1.3 & 0.8 & 0.2 & 1 & 0.64 \\
\hline Delayed recall (range: 0-4) & 1.7 & 1.4 & 0.2 & 0.3 & 27.0 & 1 & $<0.001$ \\
\hline Orientation (range: 0-6) & 5.4 & 1.0 & 4.2 & 1.0 & 18.6 & 1 & $<0.001$ \\
\hline
\end{tabular}

Bold values: $\mathrm{p}<0.05$; LLD-CI=late-life depression with cognitive impairment; $\mathrm{AD}=$ Alzheimer's disease; MoCA=Montreal Cognitive Assessment scale

\section{Associations between AD7c-NTP levels and age, gender, education and MoCA score}

Spearman correlation analyses revealed that urinary AD7c-NTP level was significantly and positively associated with the MoCA score in the
LLD-NCI group $(\mathrm{r}=0.40, \mathrm{P}=0.02)$. However, no significant associations were found in the level of urinary AD7c-NTP in relation to age, gender, education and MoCA score in the LLD-CI group.

Table 4. Associations of AD7c-NTP levels in study groups with age, gender, education, and MoCA

\begin{tabular}{lllllllll}
\hline Groups & \multicolumn{1}{c}{ Age } & \multicolumn{3}{c}{ Gender } & \multicolumn{3}{c}{ Education } & MoCA \\
\cline { 2 - 9 } & $\mathrm{r}$ & $p$ & $\mathrm{r}$ & $p$ & $\mathrm{r}$ & $p$ & $\mathrm{r}$ & $p$ \\
\hline LLD-CI $(\mathrm{n}=52)$ & 0.08 & 0.57 & 0.02 & 0.88 & 0.13 & 0.33 & 0.16 & 0.25 \\
LLD-NCI $(\mathrm{n}=29)$ & 0.27 & 0.15 & -0.25 & 0.17 & 0.09 & 0.62 & 0.40 & $\mathbf{0 . 0 2}$ \\
$\mathrm{AD}(\mathrm{n}=30)$ & -0.01 & 0.95 & 0.10 & 0.60 & 0.01 & 0.93 & -0.09 & 0.63 \\
$\mathrm{HC}(\mathrm{n}=27)$ & 0.03 & 0.87 & 0.07 & 0.67 & 0.29 & 0.11 & 0.17 & 0.36 \\
\hline Bolded values: $\mathrm{p}<0.05 ;$ LLD-CI: late-life depression with cognitive impairment; \\
LLD-NCI: late-life depression without cognitive impairment; AD: Alzheimer's \\
disease; HC: healthy control; Bolded values are p<0.05; \\
AD7c-NTP=Alzheimer-associated neuronal thread protein.
\end{tabular}

\section{Discussion}

Urinary AD7c-NTP has been proposed as a sensitive and specific diagnostic marker in early identification of probable $\mathrm{AD}$ and $\mathrm{MCI}$ [33]. According to one study, the level of urinary AD7c-NTP was $1.9 \mu \mathrm{g} / \mathrm{L}(1.9 \mathrm{ng} / \mathrm{ml})$ in mild AD, 3.9 $\mu \mathrm{g} / \mathrm{L}(3.9 \mathrm{ng} / \mathrm{ml})$ in moderate/severe $\mathrm{AD}$ and 0.6 $\mu \mathrm{g} / \mathrm{L}(0.6 \mathrm{ng} / \mathrm{ml})$ in healthy control groups [53]. The optimal cutoff value of urinary AD7c-NTP level for early diagnosis of AD was set at $1.5 \mu \mathrm{g} / \mathrm{L}(1.5 \mathrm{ng} / \mathrm{ml})$, with $90.6 \%$ sensitivity and $91.8 \%$ specificity [37].

In this study the urinary AD7c-NTP levels in LLD-CI $(1.0 \mathrm{ng} / \mathrm{ml})$ were significantly higher than those in HC $(0.5 \mathrm{ng} / \mathrm{ml})$ and LLD-NCI $(0.5 \mathrm{ng} / \mathrm{ml})$, which may be related to similar pathological changes in $\mathrm{AD}$, such as accumulation of tau protein and overexpression of AD7c-NTP [29, 54]. To date, the association between urine AD7c-NTP level and the severity of cognitive impairment has been inconclusive. Some studies found that AD7c-NTP levels were negatively related with the severity of $\mathrm{AD}$ as measured by the MMSE [32,53]. Another study also found that urinary AD7c-NTP levels in advanced $\mathrm{AD}$ were significantly higher than in early stage $\mathrm{AD}$ [37]. However, other studies found that moderate AD had a lower urinary AD7c-NTP level than mild AD [55], and several studies also failed to find any relationships between urinary AD7c-NTP levels and the severity of cognitive impairment in $\operatorname{AD}[33,53]$. Our study found a positive association between urinary AD7c-NTP level and the severity of cognitive impairment in the LLD-NCI group. However, due to the small sample size, this finding needs to be confirmed in future studies. Similar to previous findings [56, 57], our study found no association between urinary AD7c NTP level with age, gender and education.

There are several limitations to this study. First, 
the sample size was relatively small, which limits the power of the findings. In addition, due to the cross-sectional design, the dynamic changes of urinary AD7c-NTP level in the four groups could not be examined. Second, clean midstream urine specimens were collected in the morning. Instead, 24-hour urine samples should have been used because the concentration of AD7c-NTP might be influenced by diurnal fluctuations. Third, the confounding effects of depressive symptoms on cognitive performance could not be controlled for. Fourth, some variables that could influence urine AD7c-NTP levels, such as the duration of the illness and use of psychotropic medications, were not examined.

In conclusion, the level of urinary AD7c-NTP appears to be associated with cognitive impairment in LLD as it was found to be significantly higher in LLD-CI than LLD-NCI and HC subjects. Hence, urinary AD7c-NTP could serve as a potential biomarker for early identification of cognitive impairment in LLD. Prospective studies with a larger sample size are needed to confirm such findings.

\section{Acknowledgements}

The study was supported by the Beijing Municipal Administration of Hospitals Incubating Program (PX2016016) and the Beijing Municipal Administration of Hospitals' Youth Program (QML20171903).

\section{Competing Interests}

The authors have declared that no competing interest exists.

\section{References}

1. Koenig AM, Bhalla RK, Butters MA. Cognitive functioning and late-life depression. J Int Neuropsychol Soc. 2014; 20(5): 461-7.

2. Valiengo Lda C, Stella F, Forlenza OV. Mood disorders in the elderly: prevalence, functional impact, and management challenges. Neuropsychiatr Dis Treat. 2016; 12: 2105-14

3. Guerra M, Prina AM, Ferri CP, Acosta D, Gallardo S, Huang Y, et al. A comparative cross-cultural study of the prevalence of late life depression in low and middle income countries. J Affect Disord. 2016; 190: 362-8.

4. Wong MM, Chan CF, Li SW, Lau YM. Six-month Follow-up of Cognitive Impairment and Depressive Symptoms in Late-onset Depression. East Asian Arch Psychiatry. 2015; 25(4): 146-9.

5. Alexopoulos GS. Depression in the elderly. Lancet. 2005; 365: 1961-70.

6. Kessler RC, Berglund P, Demler O, Jin R, Merikangas KR, Walters EE. Lifetime prevalence and age-of-onset distributions of DSM-IV disorders in the national comorbidity survey replication. Arch Gen Psychiatry. 2005; 62(6): 593-602.

7. Baudic S, Tzortzis C, Barba GD, Traykov L. Executive deficits in elderly patients with major unipolar depression. J Geriatr Psychiatry Neurol. 2004; 17(4): 195-201

8. Butters MA, Whyte EM, Nebes RD, Begley AE, Dew MA, Mulsant BH, et al. The nature and determinants of neuropsychological functioning in late-life depression. Arch Gen Psychiatry. 2004; 61(6): 587-95.

9. Rapp MA, Dahlman K, Sano M, Grossman HT, Haroutunian V, Gorman JM. Neuropsychological differences between late-onset and recurrent geriatric major depression. Am J Psychiatry. 2005; 162(2): 691-8.

10. Ismail Z, Fischer $\mathrm{C}$, McCall WV. What characterizes late-life depression? Psychiatr Clin North Am. 2013; 36(4): 483-96.

11. Kiloh LG. Pseudo-dementia. Acta Psychiatr Scand. 1961; 37: 336-51.

12. Bhalla RK, Butters MA, Mulsant BH, Begley AE, Zmuda MD, Schoderbek B, et al. Persistence of Neuropsychologic Deficits in the Remitted State of Late-Life Depression. Am J Geriatr Psychiatry. 2006; 14(5): 419-27.
13. Kral VA, Emery OB. Long-term follow-up of depressive pseudodementia of the aged. Can J Psychiatry. 1989; 34(5): 445-6.

14. Naismith SL, Hickie IB, Turner K, Little CL, Winter V, Ward PB, et al. Neuropsychological performance in patients with depression is associated with clinical, etiological and genetic risk factors. J Clin Exp Neuropsychol. 2003; 25(6): 866-77.

15. Devanand DP, Sano M, Tang MX, Taylor S, Gurland BJ, Wilder D, et al. Depressed mood and the incidence of Alzheimer's disease in the elderly living in the community. Arch Gen Psychiatry. 1996; 53(2): 175-82.

16. Alexopoulos GS, Meyers BS, Young RC, Mattis S, Kakuma T. The course of geriatric depression with "reversible dementia": a controlled study. Am J Psychiatry. 1993; 150(11): 1693-9.

17. Steffens DC, Plassman BL, Helms MJ, Welsh-Bohmer KA, Saunders AM, Breitner JC. A twin study of late-onset depression and apolipoprotein E epsilon 4 as risk factors for Alzheimer's disease. Biol Psychiatry. 1997; 41(8): 851-6

18. Geda YE, Knopman DS, Mrazek DA, Jicha GA, Smith GE, Negash S, et al. Depression, apolipoprotein E genotype, and the incidence of mild cognitive impairment: a prospective cohort study. Arch Neurol. 2006; 63(3): 435-40.

19. Butters MA, Young JB, Lopez O, Aizenstein HJ, Mulsant BH, Reynolds III CF, et al. Pathways linking late-life depression to persistent cognitive impairment and dementia. Dialogues Clin Neurosci. 2008; 10(3): 345-57.

20. Cummings JL, Vinters HV, Cole GM, Khachaturian ZS. Alzheimer's disease Etiologies, pathophysiology, cognitive reserve, and treatment opportunities. Neurology. 1998; 51: S2-S17.

21. Morris JC, Goate A, Ashall F. Relationship of plaques and tangles to Alzheimer's disease phenotype. Pathobiology of Alzheimer's Disease. 1995: 191-223.

22. Jorm AF. History of depression as a risk factor for dementia: an updated review. Aust N Z J Psychiatry. 2001; 35(6): 776-81.

23. Ownby RL, Crocco E, Acevedo A, John V, Loewenstein D. Depression and risk for Alzheimer disease: systematic review, meta-analysis, and metaregression analysis. Arch Gen Psychiatry. 2006; 63(5): 530-8.

24. Rapp MA, Schnaider-Beeri M, Grossman HT, Sano M, Perl DP, Purohit DP, et al. Increased hippocampal plaques and tangles in patients with Alzheimer disease with a lifetime history of major depression. Arch Gen Psychiatry. 2006; 63(2): 161-7.

25. Wilkins $\mathrm{CH}$, Mathews J, Sheline YI. Late life depression with cognitive impairment: Evaluation and treatment. Clin Interv Aging. 2009; 4: 51-7.

26. Rue AL, Spar J, Hill CD. Cognitive Impairment in late-life depression: Clinical correlates and treatment implications. J Affect Disord. 1986; 11(3): 179-84

27. Korczyn AD, Halperin I. Depression and dementia. J Neurol Sci. 2009; 283: $139-42$

28. Monte S, Ghanbari K, Frey WH, Beheshti I, Averback P, Hauser SL, et al. Characterization of the AD7C-NTP cDNA expression in Alzheimer's disease and measurement of a 41-kD protein in cerebrospinal fluid. J Clin Invest. 1997; 100(12): 3093-104.

29. Munzar M, Levy S, Rush R, Averback P. Clinical study of a urinary competitve ELISA for neural thread protein in Alzheimer disease. Neurol Clin Neurophysiol. 2002; 2002(1): 2-8.

30. De La Monte SM, Wands JR. Alzheimer-associated neuronal thread protein-induced apoptosis and impaired mitochondrial function in human central nervous system-derived neuronal cells. J Neuropathol Exp Neurol. 2001; 60(2): 195-207.

31. de la Monte SM, Chen GJ, Rivera E, Wands JR. Neuronal thread protein regulation and interaction with microtubule-associated proteins in SH-Sy5y neuronal cells. Cell Mol Life Sci. 2003; 60(12): 2679-91.

32. Kahle PJ, Jakowec M, Teipel SJ, Hampel H, Petzinger GM, Di Monte DA, et al. Combined assessment of tau and neuronal thread protein in Alzheimer's disease CSF. Neurology. 2000; 54(7): 1498-504.

33. Zhang N, Zhang L, Li Y, Gordon ML, Cai L, Wang Y, et al. Urine AD7c-NTP Predicts Amyloid Deposition and Symptom of Agitation in Patients with Alzheimer's Disease and Mild Cognitive Impairment. J Alzheimers Dis. 2017; 60(1): 87-95.

34. de la Monte SM, Wands JR. The AD7c-NTP neuronal thread protein biomarker for detecting Alzheimer's disease. J Alzheimers Dis. 2001; 3(3): 345-53.

35. Ma L, Wang R, Han Y, Sheng S, Zhu J, Ji Z, et al. Development of a Novel Urine Alzheimer-Associated Neuronal Thread Protein ELISA Kit and Its Potential Use in the Diagnosis of Alzheimer's Disease. J Clin Lab Anal. 2016; 30(4): 308-14.

36. Zhang JJ, Shi SS. A literature review of AD7c-ntp as a biomarker for Alzheimer's disease. Ann Indian Acad Neurol. 2013; 16(3): 307-9.

37. Ghanbari H, Ghanbari K, Beheshti I, Munzar M, Vasauskas A, Averback P. Biochemical assay for AD7C-NTP in urine as an Alzheimer's disease marker. J Clin Lab Anal. 1998; 12(5): 285-8.

38. Zhang $\mathrm{J}$, Zhang $\mathrm{CH}$, Li RJ, Lin $\mathrm{XL}$, Chen $\mathrm{YD}$, Gao HQ, et al. Accuracy of urinary AD7c-NTP for diagnosing Alzheimer's disease: a systematic review and meta-analysis. J Alzheimers Dis. 2014; 40(1): 153-9.

39. Yi L, Yu H, Cui WZ. Follow-up study of persistent cognitive impairment of senile depression (in Chinese). Sichuan Mental Health. 2015; 3: 230-3.

40. Bell CC. DSM-IV: Diagnostic and Statistical Manual of Mental Disorders. Diagnostic and statistical manual of mental disorders: American Psychiatric Association. 1994:4189-4189.

41. Tan JP, Li N, Gao J, Wang LN, Zhao YM, Yu BC, et al. Optimal cutoff scores for dementia and mild cognitive impairment of the Montreal Cognitive 
Assessment among elderly and oldest-old Chinese population. J Alzheimers Dis. 2015; 43(4): 1403-12.

42. Wong A, Law LS, Liu W, Wang Z, Lo ES, Lau A, et al. Montreal Cognitive Assessment: One Cutoff Never Fits All. Stroke. 2015; 46(12): 3547-50.

43. McKhann G, Drachman D, Folstein M, Katzman R, Price D, Stadlan EM. Clinical diagnosis of Alzheimer's disease: report of the NINCDS-ADRDA Work Group under the auspices of Department of Health and Human Services Task Force on Alzheimer's Disease. Neurology. 1984; 34(7): 939-44.

44. Folstein MF, Folstein SE, McHugh PR. "Mini-mental state". A practical method for grading the cognitive state of patients for the clinician. J Psychiatr Res. 1975; 12: 189-98.

45. Hachinski VC, Iliff LD, Zilhka E, Du Boulay GH, McAllister VL, Marshall J, et al. Cerebral blood flow in dementia. Arch Neurol. 1975; 32: 632-7.

46. Nasreddine ZS, Phillips NA, Bedirian V, Charbonneau S, Whitehead V, Collin I, et al. The Montreal Cognitive Assessment, MoCA: a brief screening tool for mild cognitive impairment. J Am Geriatr Soc. 2005; 53: 695-9.

47. Wen HB, Zhang ZX, Niu FS, Li L. The application of Montreal cognitive assessment in urban Chinese residents of Beijing (in Chinese). Zhonghua Nei Ke Za Zhi. 2008; 47: 36-9.

48. Han B, Page EE, Stewart LM, Deford CC, Scott JG, Schwartz LH, et al. Depression and cognitive impairment following recovery from thrombotic thrombocytopenic purpura. Am J Hematol. 2015; 90: 709-14.

49. Smith T, Gildeh N, Holmes C. The Montreal Cognitive Assessment: Validity and Utility in a Memory Clinic Setting. Canadian Journal of Psychiatry. 2007; 52: 329-32.

50. Hamilton M. A rating scale for depression. J Neurol Neurosurg Psychiatry. 1960; 23: 56-62.

51. Wang XD, Wang XL, Ma H. Mental Health Rating Scales Handbook (updated edition) (in Chinese). Chinese Mental Health Journal. 1999: 196-202.

52. Hamilton M. The assessment of anxiety states by rating. Br J Med Psychol. 1959; 32(1): 50-5.

53. Zhu R, Yuan XR, Peng DT. Value of Alzheimer-associated neuronal thread protein level in urine for diagnosing Alzheimer's disease (in Chinese). Chinese Journal of Geriatrics 2012; 07: 575-7.

54. De la Monte S, Wands J. Neurodegeneration changes in primary central nervous system neurons transfected with the Alzheimer-associated neuronal thread protein gene. Cell Mol Life Sci. 2001; 58: 844-9.

55. Youn $\mathrm{YC}$, Park K-W, Han S-H, Kim S. Urine neural thread protein measurements in Alzheimer disease. J Am Med Dir Assoc. 2011; 12(5): 372-6.

56. Li YJ, Pan YR, Wang YL, Lin WJ, Zhou YP, Sun XJ, et al. The Study of AD7c-NTP Level in Urine of Elders with Alzheimer's Disease (in Chinese). Chinese Journal of Clinical Neurosciences. 2014; 22: 198-202.

57. Cai C, Zhou Y, Zhou Z, Liu J. The study of relationship of urine AD7c-NTP and plasma Hcy levels in Alzheimer's disease patients (in Chinese). Journal of Apoplexy and Nervous Diseases. 2013; 30: 520-3. 